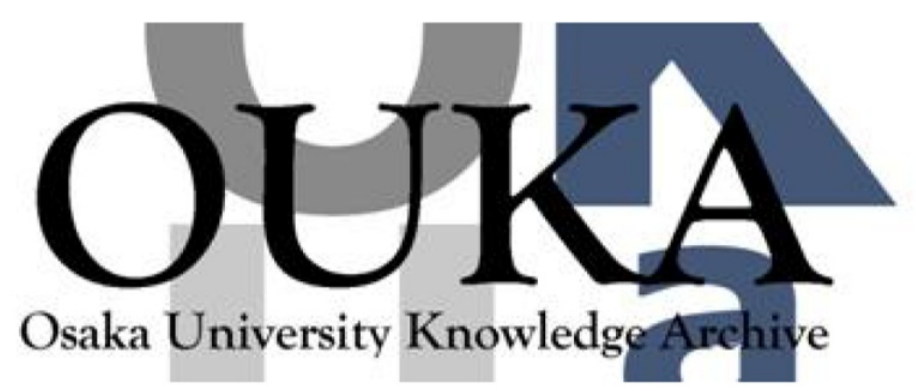

\begin{tabular}{|c|c|}
\hline Title & $\begin{array}{l}\text { Nanosurgery of sub-cellular organelles in } \\
\text { living cells using a femtosecond laser } \\
\text { oscillator }\end{array}$ \\
\hline Author (s) & $\begin{array}{l}\text { Watanabe, Wataru; Shimada, Tomoko; Matsunaga, } \\
\text { Sachihiro et al. }\end{array}$ \\
\hline Citation & $\begin{array}{l}\text { Proceedings of SPIE - The International Society } \\
\text { for Optical Engineering. } 6108 \text { p. } 610804\end{array}$ \\
\hline Issue Date & $2006-02-28$ \\
\hline oaire:version & VoR \\
\hline URL & https://hdl. handle. net/11094/79146 \\
\hline rights & $\begin{array}{l}\text { Copyright } 2006 \text { Society of Photo Optical } \\
\text { Instrumentation Engineers (SPIE). One print or } \\
\text { electronic copy may be made for personal use } \\
\text { only. Systematic reproduction and distribution, } \\
\text { duplication of any material in this publication } \\
\text { for a fee or for commercial purposes, or } \\
\text { modification of the contents of the publication } \\
\text { are prohibited. }\end{array}$ \\
\hline Note & \\
\hline
\end{tabular}

Osaka University Knowledge Archive : OUKA

https://ir. Library. osaka-u. ac. jp/

Osaka University 


\title{
Nanosurgery of sub-cellular organelles in living cells using a femtosecond laser oscillator
}

\author{
Wataru Watanabe, Tomoko Shimada, Sachihiro Matsunaga ${ }^{a}$, Hiroshi Ishii ${ }^{a}$, Tsunehito Higashi ${ }^{a}$, \\ Kiichi Fukui ${ }^{a}$, and Kazuyoshi Itoh \\ Department of Material and Life Science, Graduate School of Engineering, Osaka University \\ 2-1, Yamadaoka, Suita, Osaka 565-0871, Japan \\ ${ }^{a}$ Department of Biotechnology, Graduate School of Engineering, Osaka University \\ 2-1, Yamadaoka, Suita, Osaka 565-0871, Japan
}

\begin{abstract}
Femtosecond laser pulses in the near-infrared region have potential applications in nanosurgery in cell biology. Femtosecond laser pulses can be used to selectively disrupt and dissect intracellular organelles in living cells. We report on disruption of fluorescence-labeled nucleus and mitochondria in living HeLa cells using a femtosecond Ti:sapphire laser oscillator with a repetition rate of $76 \mathrm{MHz}$. We examined the effectiveness of the restaining method in combination with fluorescence recovery after photobleaching (FRAP) analysis to discern disruption or bleaching.
\end{abstract}

Keywords: femtosecond laser, HeLa cell, organelles, mitochondria, disruption, nanosurgery

\section{INTRODUCTION}

Near-infrared femtosecond lasers have recently attracted much attention in cell biology. Femtosecond lasers can be used to image subcellular structures using multi-photon excitation microscopy [1] without compromising viability [2-5]. Femtosecond lasers operating in the near infrared region have attractive advantages compared with conventional ultraviolet lasers, including high resolution, a background-free signal, low background scattering, deep penetration into thick samples, and reduced photon-induced damage.

Intense femtosecond lasers can also be used to perform laser nanosurgery at higher energies. Femtosecond laser surgery has become an important tool for disruption or dissection of organelles on a scale of hundreds of nanometers to micrometers. Femtosecond laser surgery has been demonstrated by use of both low-repetition-rate amplified Ti:sapphire laser systems and high-repetition-rate Ti:sapphire oscillators. Amplified systems can provide high pulse energy and cause less thermal damage due to the low repetition rate. Several groups have recently reported femtosecond laser surgery using regenerative amplifiers with pulse energies of a few nanojoules to a few tens of nanojoules, disruption of mitochondria in living cells, dissection of actin fiber bundles, ablation of nuclei in fixed endothelial cells, ablation of single microtubules in living cells, and dissection of axons [6-9].

In contrast, a femtosecond oscillator can contribute to the construction of integrated systems in combination with nonlinear imaging, including multiphoton microscopy and higher harmonic generation microscopy, as well as laser surgery. In particular, the use of a femtosecond oscillator enables laser surgery with low pulse energy in the subnanojoule region. For these reasons, several researchers have adopted femtosecond laser oscillators for nanosurgery of cells and cellular organelles [10-14]. Examples include knocking out single organelles in living cells, ablation in Drosophila embryos to induce modulation of specific movements, and the combination of two-photon microscopy and nanosurgery of fluorescent structures within yeast mitotic spindles [10-14].

In this paper, we report on the disruption of fluorescence-labeled nucleus and mitochondria in living HeLa cells by

watanabe@photonics.mls.eng.osaka-u.ac.jp; phone 81668797326 ; fax 81668794582

Commercial and Biomedical Applications of Ultrafast Lasers VI,

edited by Joseph Neev, Stefan Nolte, Alexander Heisterkamp, Christopher B. Schaffer,

Proc. of SPIE Vol. 6108, 610804, (2006) · 0277-786X/06/\$15 · doi: 10.1117/12.645474

Proc. of SPIE Vol. 6108 610804-1 
focusing femtosecond laser pulses produced by a Ti:sapphire laser oscillator. We present disruption of nucleus and mitochondria in living HeLa cells using a femtosecond Ti:sapphire laser oscillator. We examined the effectiveness of the restaining method in combination with fluorescence recovery after photobleaching (FRAP) analysis to discern disruption or bleaching.

\section{EXPERIMENTAL SETUP}

Figure 1 shows a schematic diagram of the setup used for the disruption of fluorescence-labeled organelles in living cells using a femtosecond laser oscillator [15]. The laser scanning microscope was adapted from an Olympus FV300 scanning unit combined with an Olympus IX71 inverted microscope. A collimated CW laser beam of a He-Ne laser (wavelength $543 \mathrm{~nm}$ ) or an Ar-ion laser (wavelength $488 \mathrm{~nm}$ ) was reflected by dichroic mirrors DM1 and DM2 and then focused into the cells through an oil-immersion objective lens (OB; Olympus Corporation, PlanApo60×Oil, NA 1.4). The back-propagated one-photon fluorescence was collected using the same objective lens and detected with photomultiplier tubes (PMT1 and PMT2; Hamamatsu Photonics, R928P). Bandpass filters BP1 (transmission wavelength: $510 \mathrm{~nm}-540 \mathrm{~nm}$ ) and BP2 (transmission wavelength: $560 \mathrm{~nm}-600 \mathrm{~nm}$ ) were placed before PMT1 and PMT2. Two-dimensional confocal cross-sectional images were obtained by scanning the focused laser beams in the $x y$ plane with a pair of high-speed galvanometer mirrors (GM; Cambridge, 6210) inside the laser-scanning microscope. Scanning in the depth direction ( $z$ direction) was achieved by moving the objective lens with a stepping motor to obtain three-dimensional confocal images.

Irradiation with femtosecond laser pulses was performed using a mode-locked Ti:sapphire laser oscillator with a wavelength of $800 \mathrm{~nm}$ and a repetition rate of $76 \mathrm{MHz}$ (Coherent, Mira). The laser pulses passed through a Faraday isolator (FI) to block reflections from the optical components. The laser pulses then passed through a series of SF10 prisms (P1, P2) to compensate for the dispersion of the optical components in the light path and the microscope. To fill the aperture of the objective lens (OB), the beam was reduced to $2 \mathrm{~mm}$ in diameter with a pair of lenses (L1, L2), and it was then directed onto a pair of GM via DM1 and DM2. Femtosecond laser pulses were focused into the cells through the oil-immersion objective.

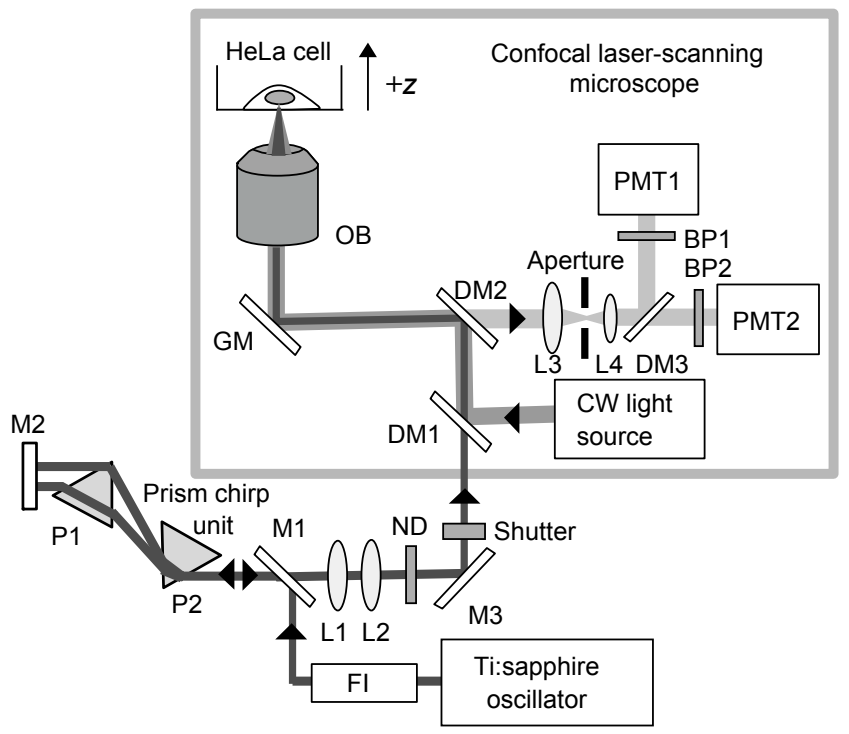

Figure 1: Schematic diagram of the experimental setup. FI, Faraday isolator; P, SF10 prism; M mirror; L, lens; ND, neutral density filter; DM, dichroic mirror; OB, objective lens; GM, pair of galvanometer mirrors; PMT, photomultiplier tube. 


\section{DISRUPTION OF NUCLEUS}

We examined the effectiveness of the restaining method in combination with fluorescence recovery after photobleaching (FRAP) analysis to discern disruption or bleaching.. Here, we used the enhanced green fluorescent protein (EGFP) labeled nucleus [15]. Figure 2 shows FRAP analysis of the EGFP labeled nucleus at different energies. An EGFP labeled nuclear region of $1.5 \times 1.5 \mu \mathrm{m}^{2}$ in a living HeLa cell was irradiated while varying the femtosecond laser energy $(0.21,0.26$, and $0.39 \mathrm{~nJ} /$ pulse) at a wavelength of $925 \mathrm{~nm}$. An exposure time was $1.1 \mathrm{~s}$ for scanning the nuclear region of $1.5 \times 1.5 \mu \mathrm{m}^{2}$. Time-lapse images of the nucleus were acquired by excitation with the $\mathrm{Ar}^{+}$laser before and after femtosecond laser irradiation. At the energies of 0.21 and $0.26 \mathrm{~nJ} / \mathrm{pulse}$, the fluorophore was bleached, and subsequent recovery of fluorescence in the bleached region occurred due to inward diffusion of unbleached fluorophore molecules. Figure 3 shows relative fluorescence intensity of the bleached area after femtosecond laser irradiation. The time required for $50 \%$ recovery of fluorescence was $20 \mathrm{~s}$ at the energies of 0.21 and $0.26 \mathrm{~nJ} /$ pulse. Figures 2 and 3 show that at the energy of $0.39 \mathrm{~nJ} /$ pulse, fluorescence recovery was not observed where fluorescence in the focal region disappeared.
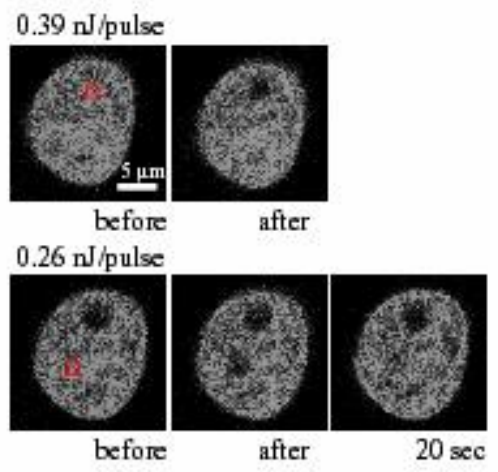

$0.21 \mathrm{~nJ} /$ pulse

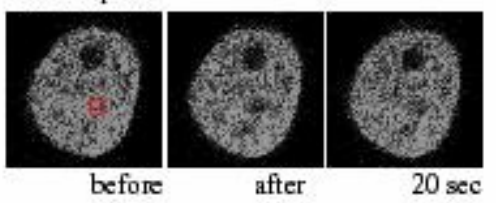

Figure 2: FRAP analysis of the enhanced green fluorescent protein (EGFP) labeled nucleus at different energies.

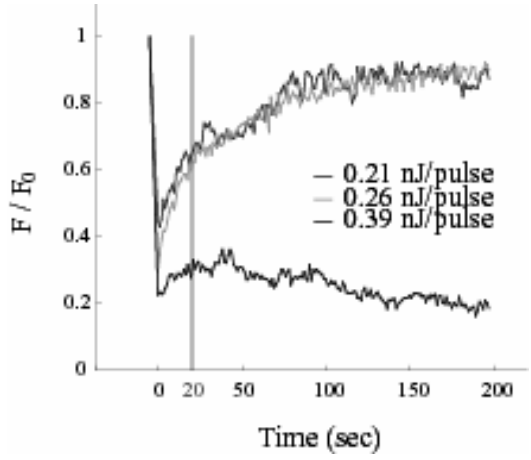

Figure 3: Relative fluorescence intensity of the bleached area. 
After femtosecond laser irradiation, we restained the same cell with a blue fluorophore (Hoechest 33342). Because nuclei normally became suitably stained $40 \mathrm{~min}$ after the addition of Hoechest 33342 , we obtained fluorescence images after 45 minutes. Figure 4(a) and (b) show fluorescence images of the nuclei labeled with EGFP and that stained with Hoechest 33342 in the restaining experiments. When the bleaching occurred, fluorescence was also observed from both EGFP and Hoechest 33342. When disruption of the nucleus occurred, no fluorescence was observed from EGFP or Hoechest 33342 in the laser-irradiated region. These results demonstrate that observation of fluorescence recovery is an indicator of the disruption of organelles in living cells.

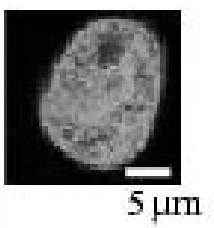

GFP

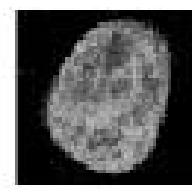

Hoechest 33342

(a)

(b)

Figure 4: Restaining experiments. (a) Fluorescence image of EGFP. (b) Fluorescence image of Hoechest 33342.

\section{DISRUPTION OF MITOCHONDRIA}

We show the disruption of individual mitochondria in living HeLa cells using the femtosecond laser oscillator system described above [15]. The cells expressed the enhanced yellow fluorescent protein (EYFP) in the mitochondria. The cells were transfected with an EYFP fused with a mitochodria-targeted sequence of cytochrome C oxidase. Stacked three-dimensional confocal images of cells were observed by excitation with the $\mathrm{Ar}^{+}$laser, as shown in Fig. 5(a). To produce a stacked 3D image, nine confocal cross-sectional images were obtained by translating the objective lens by 2 $\mu \mathrm{m}$ in the depth $(z)$ direction in steps of $250 \mathrm{~nm}$. A train of femtosecond laser pulses with an energy of $0.39 \mathrm{~nJ} / \mathrm{pulse}$ was focused at a spot and the shutter was open for an exposure time of $32 \mathrm{~ms}$, which corresponded to $2.4 \times 10^{6}$ pulses. The time duration necessary to obtain two stacked confocal images before and after femtosecond laser irradiation was 30 seconds. Figure 5(b) shows the stacked confocal image obtained after femtosecond laser irradiation. Figures 5 show that fluorescence from a single mitochondrion within a size of a few microns disappeared. Displacement of the mitochondria outside the focal region between the before and after images was attributed to cytoplasmic streaming, which indicated the viability of the cells.

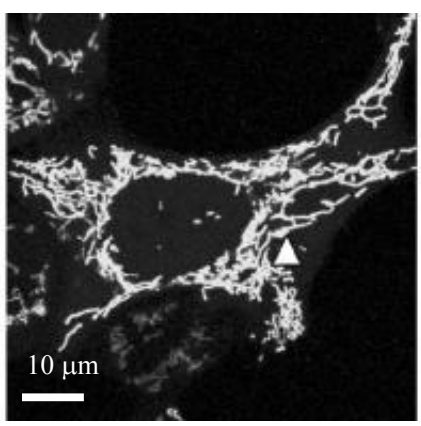

(a)

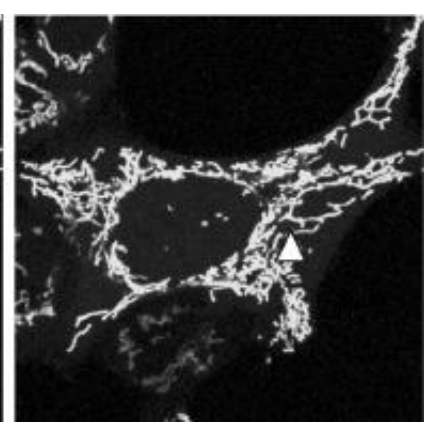

(b)

Figure 5: Stacked three-dimensional confocal images (a) before and (b) after femtosecond laser irradiation. Fluorescence image shows mitochondria of HeLa cells visualized by EYFP. A target mitochondrion is indicated by an arrow. 
We confirmed disruption of the mitochondrion using the restaining method. The EYFP in the mitochondria onto which the femtosecond laser pulses were focused was not restained with MitoTracker Red. Previous FRAP experiments on mitochondria showed that the time required for $50 \%$ recovery of fluorescence was a few seconds [16]. Since the mitochondria irradiated by the femtosecond laser pulses in our experiments did not exhibit fluorescence recovery after $30 \mathrm{~s}$, the mechanism must be different from bleaching. We have therefore shown that disruption and bleaching are distinguishable using FRAP analysis and the restaining method.

\section{CONCLUSION}

We present femtosecond laser disruption of organelles using a Ti:sapphire laser oscillator with a repetition rate of 76 MHz. Disruption of fluorescence-labeled nucleus and mitochondria in living HeLa cells with focused femtosecond laser pulses was experimentally demonstrated. We demonstrated the effectiveness of the restaining method in combination with fluorescence recovery after photobleaching (FRAP) analysis to discern disruption or bleaching.

\section{REFERENCES}

1. W. Denk, J. H. Strickler, and W. W. Webb, “Two-photon laser scanning fluorescence microscopy," Science 248, pp. 73-76, 1990.

2. K. König, P. T. C. So, W. W. Mantulin, and E. Gratton, "Cellular response to near-infrared femtosecond laser pulses in two-photon microscopes," Opt. Lett. 22, pp. 135-136, 1997.

3. K. König, T. W. Becker, P. Fischer, I. Riemann, and K. -J. Halbhuber, "Pulse-length dependence of cellular response to intense near-infrared laser pulses in multiphoton microscopes," Opt. Lett. 24, pp. 113-11, 1999.

4. J. M. Squirrell, D. L. Wokosin, J. G. White, and B. D. Bavister, "Long-term two-photon fluorescence imaging of mammalian embryos without compromising viability," Nat. Biotechnol. 17, pp. 763-767, 1999.

5. V. V. Yakovlev, “Advanced instrumentation for non-linear Raman microscopy," J. Raman Spectrosc. 4, pp. $957-$ 964, 2003.

6. W. Watanabe, N. Arakawa, S. Matsunaga, T. Higashi, K. Fukui, K. Isobe, and K. Itoh, "Femtosecond laser disruption of subcellular organelles in a living cell," Opt. Express 12, pp. 4203-4213, 2004.

7. N. Shen, D. Datta, C. B. Schaffer, P. LeDuc, D. E. Ingber, and E. Mazur, "Ablation of cytoskeletal filaments and mitochondria in live cells using a femtosecond laser nanoscissor," Mech. Chem. Biosyst. 2, pp. 17-25, 2005.

8. A. Heisterkamp, I. Z. Maxwell, E. Mazur, J. M. Underwood, J. A. Nickerson, S. Kumar, and D. E. Ingber, "Pulse energy dependence of subcellular dissection by femtosecond laser pulses," Opt. Express 13, pp. 3690-3696, 2005.

9. M. F. Yanik, H. Cinar, H. N. Cinar, A. D. Chisholm, Y. Jin, and A. Ben-Yakar, "Functional regeneration after laser axotomy," Nature 432, p. 822, 2004.

10. K. König, "Laser tweezers and multiphoton microscopes in life sciences," Histochem. Cell Biol. 114, pp. 79-92, 2000.

11. K. König, I. Riemann, and W. Fritzsche, "Nanodissection of human chromosomes with near-infrared femtosecond laser pulses," Opt. Lett. 26, pp. 819-821, 2001.

12. U. K. Tirlapur and K. König, "Targeted transfection by femtosecond laser," Nature 418, pp. 290-291, 2002.

13. W. Supatto, D. Débarre, B. Moulia, E. Brouzés, J. -L. Martin, E. Farge, and E. Beaurepaire, "In vivo modulation of morphogenetic movements in Drosophila embryos with femtosecond laser pulses," Proc. Natl. Acad. Sci. USA 102, pp. 1047-1052, 2005.

14. L. Sacconi, I. M. Tolić-Nørrelykke, R. Antolini, and F. S. Pavone, "Combined intracellular three-dimensional imaging and selective nanosurgery by a nonlinear microscope," J. Biomed. Opt. 10, p. 014002, 2005.

15. T. Shimada, W. Watanabe, S. Matsunaga, T. Higashi, H. Ishii, K. Fukui, K. Isobe, and K. Itoh, "Intracellular disruption of mitochondria in living HeLa cells with a 76-MHz femtosecond laser oscillator," Opt. Express 13, pp. 9869-9880, 2005.

16. T. J. Collins and M. D. Bootman, "Mitochondria are morphologically heterogeneous within cells," J. Exp. Biol. 206, pp. 1993-2000, 2003. 